Pacific

Journal of

Mathematics

SOME SUMMATIONS OF $q$-SERIES BY TELESCOPING

M.V. Subbarao and A. Verma 


\title{
SOME SUMMATIONS OF $q$-SERIES BY TELESCOPING
}

\author{
M.V. Subbarao AND A. Verma
}

\begin{abstract}
Summation formulae for $q$-series with independent bases are obtained and used to derive transformation and expansion of $q$-series involving independent bases.
\end{abstract}

\section{Introduction.}

The sum of the first $(n+1)$-terms of the non-terminating very-well-poised ${ }_{6} \phi_{5}[q][1]$

$$
\sum_{k=0}^{n} \frac{\left(1-a q^{2 k}\right)}{(1-a)} \frac{\left(a, b, c, \frac{a}{b c} ; q\right)_{k}}{\left(q, \frac{a q}{b}, \frac{a q}{c}, b c q ; q\right)_{k}} q^{k}=\frac{\left(a q, b q, c q, \frac{a q}{b c} ; q\right)_{n}}{\left(\frac{a q}{b}, \frac{a q}{c}, b c q, q ; q\right)_{n}}
$$

follows from Jacksons $q$-analgue of Whipple's summation formula for a terminating very-well-poised balanced ${ }_{8} \phi_{7}[q]$ (in $[4,2.6 .2]$ setting $e=a q^{n+1}$ ). A bibasic analogue of (1.1) was obtained by Gasper [3].

$$
\begin{aligned}
& \sum_{k=0}^{n} \frac{\left(1-a q^{k} p^{k}\right)\left(1-b p^{k} q^{-k}\right)}{(1-a)(1-b)} \frac{(a, b ; p)_{k}\left(c, \frac{a}{b c} ; q\right)_{k}}{\left(q, \frac{a q}{b}, q\right)_{k}\left(\frac{a p}{c}, b c p ; p\right)_{k}} q^{k} \\
& =\frac{(a p, b p ; p)_{n}\left(c q, \frac{a q}{b c} ; q\right)_{n}}{\left(q, \frac{a q}{b} ; q\right)_{n}\left(\frac{a p}{c}, b c p ; p\right)_{n}}, \quad n=0,1,2, \ldots
\end{aligned}
$$

and he used it for obtaining quadratic and cubic summation and transformations formulae for $q$-hypergeometric series. A little later Gasper and Rahman [5] obtained a bilateral extension of Gasper's bibasic summation formula (1.2):

If $m, n$ are non-negative integers, then

$$
\begin{aligned}
& \sum_{k=-m}^{n} \frac{\left(1-a d p^{k} q^{k}\right)}{(1-a d)} \frac{\left(1-\frac{b p^{k} q^{-k}}{d}\right)}{\left(1-\frac{b}{d}\right)} \frac{(a, b ; p)_{k}}{\left(d q, \frac{a d q}{b} ; q\right)_{k}} \frac{\left(c, \frac{a d^{2}}{b c} ; q\right)_{k}}{\left(\frac{a d p}{c}, \frac{b c p}{d} ; p\right)_{k}} q^{k} \\
& =\frac{(1-a)(1-b)(1-c)\left(1-\frac{a d^{2}}{b c}\right)}{d(1-a d)\left(1-\frac{b}{d}\right)\left(1-\frac{c}{d}\right)\left(1-\frac{a d}{b c}\right)} \\
& \times\left\{\frac{(a p, b p ; p)_{n}}{\left(d q, \frac{a d q}{b} ; q\right)_{n}} \frac{\left(c q, \frac{a d^{2} q}{b c} ; q\right)_{n}}{\left(\frac{a d p}{c}, \frac{b c p}{d} ; q\right)_{n}}-\frac{\left(\frac{c}{a d}, \frac{d}{b c} ; p\right)_{m+1}\left(\frac{1}{d}, \frac{b}{a d} ; q\right)_{m+1}}{\left(\frac{1}{c}, \frac{b c}{a d^{2}} ; q\right)_{m+1}\left(\frac{1}{a}, \frac{1}{b} ; p\right)_{m+1}}\right\} .
\end{aligned}
$$


Jain and Verma [6] used transformations of $q$-hypergeometric series to obtain a summation formula involving three independent bases:

$$
\begin{aligned}
& \sum_{k=-m}^{n} \frac{(\beta ; p)_{k}(c ; q)_{k}(y ; p)_{k}\left(\frac{\beta y c}{d^{2}} ; \frac{p P}{q}\right)_{k}\left[\left(1-\frac{\beta c y}{d} p^{k} P^{k}\right)\left(1-\frac{y}{d} P^{k} q^{-k}\right)\left(1-\frac{\beta}{d} p^{k} q^{-k}\right)\right] q^{k}}{(d q ; q)_{k}\left(\frac{\beta c p}{d} ; p\right)_{k}\left(\frac{\beta y}{d} \frac{p P}{q} ; \frac{p P}{q}\right)_{k}\left(\frac{c y P}{d^{2}} ; P\right)_{k}} \\
& =\frac{(1-\beta)(1-c)(1-y)\left(1-\frac{\beta c y}{d^{2}}\right)}{(c-d)}\left\{\frac{\left(\frac{d}{\beta c} ; p\right)_{m+1}\left(\frac{1}{d} ; q\right)_{m+1}\left(\frac{d}{c y} ; P\right)_{m+1}\left(\frac{d}{\beta y} ; \frac{p P}{q}\right)_{m+1}}{\left(\frac{1}{\beta} ; p\right)_{m+1}\left(\frac{1}{c} ; q\right)_{m+1}\left(\frac{1}{y} ; P\right)_{m+1}\left(\frac{d^{2}}{\beta c y} ; \frac{p P}{q}\right)_{m+1}}\right. \\
& \left.-\frac{(\beta p ; p)_{n}(c q ; q)_{n}(y P ; P)_{n}\left(\frac{\beta c y}{d^{2}} \frac{p P}{q} ; \frac{p P}{q}\right)_{n}}{\left(\frac{\beta c p}{d} ; p\right)_{n}(d q ; q)_{n}\left(\frac{c y P}{d} ; P\right)_{n}\left(\frac{\beta y}{d} \frac{p P}{q} ; \frac{p P}{q}\right)_{n}}\right\},
\end{aligned}
$$

which for $P=q$ reduces to the Gasper-Rahman's summation formula (1.3). The proof of (1.4) could be given by considering

$$
\beta_{k}=\frac{(\beta p ; p)_{k}(c q ; q)_{k}(y P ; P)_{k}\left(\frac{\beta c y}{d^{2}} \frac{p P}{q} ; \frac{p P}{q}\right)_{k}}{\left(\frac{\beta c}{d} p ; p\right)_{k}(d q ; q)_{k}\left(\frac{c y}{d} P ; P\right)_{k}\left(\frac{\beta y}{d} \frac{p P}{q} ; \frac{p P}{q}\right)_{k}}
$$

and observing that

$$
\begin{aligned}
\Delta \beta_{k}= & (\beta p ; p)_{k-1}(c q ; q)_{k-1}(y P ; P)_{k-1}\left(\frac{\beta y c}{d^{2}} \frac{p P}{q} ; \frac{p P}{q}\right)_{k-1} \\
& \times \frac{\left[\left(1-\frac{\beta c y}{d^{2}} p^{k} P^{k}\right)\left(1-\frac{y}{d} P^{k} q^{-k}\right)\left(1-\frac{\beta}{d} p^{k} q^{-k}\right)\right]}{\left(\frac{\beta c p}{d} ; p\right)_{k}(d q ; q)_{k}\left(\frac{\beta y}{d} \frac{p P}{q} ; \frac{p P}{q}\right)_{k}\left(\frac{c y P}{d} ; P\right)_{k}},
\end{aligned}
$$

and summing for $k$ from $-m$ to $n$ ( $m, n$ are non-negative integers) and using the usual convention:

$$
\prod_{k=m}^{n} A_{k}= \begin{cases}A_{m} A_{m+1} \cdots A_{n} & m \leq n \\ 1 & m=n-1 \\ \left(A_{n+1} A_{n+2} \cdots A_{m-1}\right)^{-1} & m \geq n-2 .\end{cases}
$$

Chu [2] obtained a generalization of Gasper-Rahman's formula (after renaming suitably the sequences so as to remove redundant sequences)

$$
\begin{aligned}
& \sum_{k=-m}^{n} \frac{\left(1-\alpha a_{k} b_{k}\right)\left(b_{k}-\frac{a_{k}}{\alpha d}\right)}{\left(1-\alpha a_{0} b_{0}\right)\left(b_{0}-\frac{a_{0}}{d \alpha}\right)} \frac{\prod_{j=0}^{k-1}\left[\left(1-a_{j}\right)\left(1-\frac{a_{j}}{d}\right)\left(1-c b_{j}\right)\left(1-\frac{\alpha^{2} d}{c} b_{j}\right)\right]}{\prod_{j=1}^{k}\left[\left(1-\alpha b_{j}\right)\left(1-\alpha d b_{j}\right)\left(1-\frac{\alpha a_{j}}{c}\right)\left(1-\frac{c}{d \alpha} a_{j}\right)\right]} \\
& =\frac{\left(1-a_{0}\right)\left(1-\frac{a_{0}}{d}\right)\left(1-b_{0} c\right)\left(1-\frac{\alpha^{2} d}{c} b_{0}\right)}{\alpha\left(1-\alpha a_{0} b_{0}\right)\left(1-\frac{c}{\alpha}\right)\left(b_{0}-\frac{a_{0}}{d \alpha}\right)\left(1-\frac{\alpha d}{c}\right)}
\end{aligned}
$$




$$
\begin{aligned}
& \times\left\{\prod_{j=1}^{n}\left[\frac{\left(1-a_{j}\right)\left(1-\frac{a_{j}}{d}\right)\left(1-b_{j} c\right)\left(1-\frac{\alpha^{2} d}{c} b_{j}\right)}{\left(1-\alpha b_{j}\right)\left(1-\alpha b_{j} d\right)\left(1-\frac{\alpha a_{j}}{c}\right)\left(1-\frac{c}{\alpha d} a_{j}\right)}\right]\right. \\
& \left.-\prod_{j=-m}^{0}\left[\frac{\left(1-\alpha b_{j}\right)\left(1-\alpha d b_{j}\right)\left(1-\frac{\alpha a_{j}}{c}\right)\left(1-\frac{c}{\alpha d} a_{j}\right)}{\left(1-a_{j}\right)\left(1-\frac{a_{j}}{d}\right)\left(1-c b_{j}\right)\left(1-\frac{\alpha^{2} d}{c} b_{j}\right)}\right]\right\}
\end{aligned}
$$

where $\left\langle a_{j}\right\rangle$ and $\left\langle b_{j}\right\rangle$ are arbitrary sequences such that none of the terms in the denominators vanish. This reduces to the Gasper-Rahman summation formula on setting $a_{k}=a p^{k}, b_{k}=q^{k}$ and replacing $\alpha$ and $d$ by $d$ and $a / b$, respectively.

In this paper we obtain in $\S 2$ a generalization of Chu's summation formula (1.7) involving four arbitrary sequences, which on specialization yields an extension of (1.1) to a summation formula with four independent bases $p, q, P$ and $Q$ and incorporating (1.7) as a special case. An expansion of the series $\sum_{n=0}^{\infty} A_{n} B_{n}(w x)^{n}$ into a series involving three independent bases is developed. A transformation of a series involving eight independent sequences is also developed. The note is concluded by obtaining in $\S 3$ some summation formulas which are different from the known ones by telescoping of series including $q$-Paff-Saalschütz's summation formula for a terminating balanced ${ }_{3} \phi_{2}[q]$.

\section{$\S 2$.}

We begin this section by proving the summation formula:

If $\left\langle u_{k}\right\rangle,\left\langle v_{k}\right\rangle,\left\langle w_{k}\right\rangle$ and $\left\langle z_{k}\right\rangle$ are arbitrary sequences such that none of the terms in the denominators vanish and $M, N$ are non-negative integers then

$$
\begin{aligned}
& \sum_{k=-m}^{n} \frac{\left(1-u_{k} v_{k} w_{k} z_{k}\right)\left(1-\frac{w_{k} z_{k}}{u_{k} v_{k}}\right)\left(1-\frac{v_{k} z_{k}}{u_{k} w_{j}}\right)\left(1-\frac{u_{k} z_{k}}{v_{k} w_{k}}\right)}{\left(1-u_{0} v_{0} w_{0} z_{0}\right)\left(1-\frac{w_{0} z_{0}}{u_{0} v_{0}}\right)\left(1-\frac{v_{0} z_{0}}{u_{0} w_{0}}\right)\left(1-\frac{u_{0} z_{0}}{v_{0} w_{0}}\right)} \\
& \quad \times \frac{\prod_{j=0}^{k-1}\left[\left(1-u_{j}^{2}\right)\left(1-v_{j}^{2}\right)\left(1-w_{j}^{2}\right)\left(1-z_{j}^{2}\right)\right]\left(\frac{u_{k} v_{k} w_{k}}{z_{k}}\right)}{\prod_{j=1}^{k}\left[\left(1-\frac{v_{j} w_{j} z_{j}}{u_{j}}\right)\left(1-\frac{u_{j} w_{j} z_{j}}{v_{j}}\right)\left(1-\frac{u_{j} v_{j} z_{j}}{w_{j}}\right)\left(1-\frac{u_{j} v_{j} w_{j}}{z_{j}}\right)\right]} \\
& =\frac{\left(1-u_{0}^{2}\right)\left(1-v_{0}^{2}\right)\left(1-w_{0}^{2}\right)\left(1-z_{0}^{2}\right)}{\left(1-u_{0} v_{0} w_{0} z_{0}\right)\left(1-\frac{w_{0} z_{0}}{u_{0} v_{0}}\right)\left(1-\frac{v_{0} z_{0}}{u_{0} w_{0}}\right)\left(1-\frac{u_{0} z_{0}}{v_{0} w_{0}}\right)} \\
& \quad \times\left\{\prod_{j=1}^{n}\left[\frac{\left(1-u_{j}^{2}\right)\left(1-v_{j}^{2}\right)\left(1-w_{j}^{2}\right)\left(1-z_{j}^{2}\right)}{\left(1-\frac{v_{j} w_{j} z_{j}}{u_{j}}\right)\left(1-\frac{u_{j} w_{j} z_{j}}{v_{j}}\right)\left(1-\frac{u_{j} v_{j} z_{j}}{w_{j}}\right)\left(1-\frac{u_{j} v_{j} w_{j}}{z_{j}}\right)}\right]\right.
\end{aligned}
$$




$$
\left.-\prod_{j=-m}^{0}\left[\frac{\left(1-\frac{v_{j} w_{j} z_{j}}{u_{j}}\right)\left(1-\frac{u_{j} w_{j} z_{j}}{v_{j}}\right)\left(1-\frac{u_{j} v_{j} z_{j}}{w_{j}}\right)\left(1-\frac{u_{j} v_{j} w_{j}}{z_{j}}\right)}{\left(1-u_{j}^{2}\right)\left(1-v_{j}^{2}\right)\left(1-w_{j}^{2}\right)\left(1-z_{j}^{2}\right)}\right]\right\} .
$$

Proof. Let

$$
\tau_{k}=\prod_{j=1}^{k}\left[\frac{\left(1-u_{j}^{2}\right)\left(1-v_{j}^{2}\right)\left(1-w_{j}^{2}\right)\left(1-z_{j}^{2}\right)}{\left(1-\frac{v_{j} w_{j} z_{j}}{u_{j}}\right)\left(1-\frac{u_{j} w_{j} z_{j}}{v_{j}}\right)\left(1-\frac{u_{j} v_{j} z_{j}}{w_{j}}\right)\left(1-\frac{u_{j} v_{j} w_{j}}{z_{j}}\right)}\right] .
$$

Then by straight forward calculations, we get

$$
\begin{aligned}
& \Delta \tau_{k}= \frac{\prod_{j=1}^{k-1}\left[\left(1-u_{j}^{2}\right)\left(1-v_{j}^{2}\right)\left(1-w_{j}^{2}\right)\left(1-z_{j}^{2}\right)\right]}{\prod_{j=1}^{k}\left[\left(1-\frac{v_{j} w_{j} z_{j}}{u_{j}}\right)\left(1-\frac{u_{j} w_{j} z_{j}}{v_{j}}\right)\left(1-\frac{u_{j} v_{j} z_{j}}{w_{j}}\right)\left(1-\frac{u_{j} v_{j} w_{j}}{z_{j}}\right)\right]\left(\frac{z_{k}}{u_{k} v_{k} w_{k}}\right)} \\
& \times\left[\left(1-u_{k} v_{k} w_{k} z_{k}\right)\left(1-\frac{w_{k} z_{k}}{u_{k} v_{k}}\right)\left(1-\frac{v_{k} z_{k}}{u_{k} w_{k}}\right)\left(1-\frac{u_{k} z_{k}}{v_{k} w_{k}}\right)\right]
\end{aligned}
$$

where $\Delta \tau_{k}=\tau_{k}-\tau_{k-1}$. Now summing with respect to $k$ from $-m$ to $n$, and using the fact that $\sum_{k=-m}^{n} \tau_{k}=\tau_{n}-\tau_{-m-1}$ and keeping in mind (1.6), we get (2.1) on simplification.

By setting $u_{j}=\sqrt{a} p^{j}, v_{j}=\sqrt{c} q^{j}, w_{j}=\sqrt{b} P^{j}, z_{j}=d \sqrt{\frac{a}{b c}} Q^{j}$ in (2.1), we get a summation formula involving four independent bases:

$$
\begin{aligned}
\sum_{k=-m}^{n} \frac{\left(1-a d p^{k} q^{k} P^{k} Q^{k}\right)\left(c-\frac{d P^{k} Q^{k}}{p^{k} q^{k}}\right)\left(1-\frac{b p^{k} P^{k}}{d q^{k} Q^{k}}\right)\left(1-\frac{a d}{b c} \frac{p^{k} Q^{k}}{q^{k} P^{k}}\right) q^{2 k}}{(1-a d)(c-d)\left(1-\frac{b}{d}\right)\left(1-\frac{a d}{b c}\right)} \\
\quad \times \frac{\left(a ; p^{2}\right)_{k}\left(c ; q^{2}\right)_{k}\left(b ; P^{2}\right)_{k}\left(\frac{a d^{2}}{b c} ; Q^{2}\right)_{k}}{\left(d \frac{q P Q}{p} ; \frac{q P Q}{p}\right)_{k}\left(\frac{a d}{c} \frac{p P Q}{q} ; \frac{p P Q}{q}\right)_{k}\left(\frac{a d}{b} \frac{p q Q}{P} ; \frac{p q Q}{P}\right)_{k}\left(\frac{b c}{d} \frac{p q P}{Q} ; \frac{p q P}{Q}\right)_{k}} \\
=\frac{(1-a)(1-b)(1-c)\left(1-\frac{a d^{2}}{b c}\right)}{(1-a d)(c-d)\left(1-\frac{b}{d}\right)\left(1-\frac{a d}{b c}\right)} \\
\quad \times\left\{\frac{\left(a p^{2} ; p^{2}\right)_{n}\left(c q^{2} ; q^{2}\right)_{n}\left(b P^{2} ; P^{2}\right)_{n}\left(\frac{a d^{2}}{b c} Q^{2} ; Q^{2}\right)_{n}}{\left(\frac{d q P Q}{p} ; \frac{q P Q}{p}\right)_{n}\left(\frac{a d}{c} \frac{p P Q}{q} ; \frac{p P Q}{q}\right)_{n}\left(\frac{a d}{b} \frac{p q Q}{P} ; \frac{p q Q}{P}\right)_{n}\left(\frac{b c}{d} \frac{p q P}{Q} ; \frac{p q P}{Q}\right)_{n}}\right. \\
\left.\quad-\frac{\left(\frac{1}{d} ; \frac{q P Q}{p}\right)_{m+1}\left(\frac{c}{a d} ; \frac{p P Q}{q}\right)_{m+1}\left(\frac{b}{a d} ; \frac{p q Q}{P}\right)_{m+1}\left(\frac{d}{b c} ; \frac{p q P}{Q}\right)_{m+1}}{\left(\frac{1}{a} ; p^{2}\right)_{m+1}\left(\frac{1}{c} ; q^{2}\right)_{m+1}\left(\frac{1}{b} ; P^{2}\right)_{m+1}\left(\frac{b c}{a d} ; Q^{2}\right)_{m+1}}\right\} .
\end{aligned}
$$

Summation formula (2.2), on setting $Q=\frac{p P}{q}$ and replacing $p^{2}, q^{2}, P^{2}, a, b$, $d$ by $p, q, P, \beta, y, \frac{c y}{d}$, respectively, reduces to the summation formula (1.4), which in turn incorporates (1.3) and (1.2) as special cases. 
It may be pointed out that (2.2) reduces to Chu's summation formula (1.7) on setting $u_{j}=\sqrt{a_{j}}, v_{j}=\sqrt{a_{j}} / \sqrt{d}, w_{j}=\sqrt{c b_{j}}, z_{j}=\alpha \sqrt{d / c} \sqrt{b_{j}}$.

Setting $m=0$ in (2.2), replacing $z_{i}$ by $\alpha z_{i}$ and setting $\alpha=\frac{u_{0}}{v_{0} w_{0} z_{0}}$, we get that

$$
\begin{aligned}
& \sum_{j=0}^{n} \frac{\left(\frac{v_{0} w_{0} z_{0}}{u_{0}}-u_{j} v_{j} w_{j} z_{j}\right)\left(\frac{v_{0} w_{0} z_{0}}{u_{0}}-\frac{w_{j} z_{j}}{u_{j} v_{j}}\right)\left(\frac{v_{0} w_{0} z_{0}}{u_{0}}-\frac{v_{j} z_{j}}{u_{j} w_{j}}\right)\left(\frac{v_{0} w_{0} z_{0}}{u_{0}}-\frac{u_{j} z_{j}}{v_{j} w_{j}}\right) \frac{u_{j} v_{j} w_{j}}{z_{j}}}{\left(1-u_{0}^{2}\right)\left(1-v_{0}^{2}\right)\left(1-w_{0}^{2}\right)\left(v_{0}^{2} w_{0}^{2}-u_{0}^{2}\right)} \\
& \times \frac{\prod_{i=0}^{j-1}\left[\left(1-u_{i}^{2}\right)\left(1-v_{i}^{2}\right)\left(1-w_{i}^{2}\right)\left(\frac{v_{0}^{2} w_{0}^{2} z_{0}^{2}}{u_{0}^{2}}-z_{i}^{2}\right)\right]}{\prod_{i=1}^{k}\left[\left(\frac{v_{0} w_{0} z_{0}}{u_{0}}-\frac{v_{i} w_{i} z_{i}}{u_{i}}\right)\left(\frac{v_{0} w_{0} z_{0}}{u_{0}}-\frac{u_{i} w_{i} z_{i}}{v_{i}}\right)\left(\frac{v_{0} w_{0} z_{0}}{u_{0}}-\frac{u_{i} v_{i} z_{i}}{w_{i}}\right)\left(\frac{u_{0}}{v_{0} w_{0} z_{0}}-\frac{u_{i} v_{i} w_{i}}{z_{i}}\right)\right]} \\
& =\frac{z_{0}^{3}}{v_{0} w_{0} u_{0}^{3}} \\
& \times \prod_{i=1}^{n}\left[\frac{\left(1-u_{i}^{2}\right)\left(1-v_{i}^{2}\right)\left(1-w_{i}^{2}\right)\left(\frac{v_{0}^{2} w_{0}^{2} z_{0}^{2}}{u_{0}^{2}}-z_{i}^{2}\right)}{\left.\left(\frac{v_{o} w_{0} z_{0}}{u_{0}}-\frac{v_{i} w_{i} z_{i}}{u_{i}}\right)\left(\frac{v_{0} w_{0} z_{0}}{u_{0}}-\frac{u_{i} w_{i} z_{i}}{v_{i}}\right)\left(\frac{v_{0} w_{0} z_{0}}{u_{0}}-\frac{u_{i} v_{i} z_{i}}{w_{i}}\right)\left(\frac{u_{0}}{v_{0} w_{0} z_{0}}-\frac{u_{i} v_{i} w_{i}}{z_{i}}\right)\right]}\right] .
\end{aligned}
$$

Next, using (2.3), the following transformation involving eight arbitrary sequences is obtained:

$$
\begin{aligned}
& \sum_{k=0}^{n} \prod_{i=1}^{n-k}\left[\frac{\left(1-U_{i}^{2}\right)\left(1-V_{i}^{2}\right)\left(1-W_{i}^{2}\right)\left(\frac{V_{0}^{2} W_{0}^{2} Z_{0}^{2}}{U_{0}^{2}}-Z_{i}^{2}\right)}{\left(\frac{V_{0} W_{0} Z_{0}}{U_{0}}-\frac{V_{i} W_{i} Z_{i}}{U_{i}}\right)\left(\frac{V_{0} W_{0} Z_{0}}{U_{0}}-\frac{U_{i} W_{i} Z_{i}}{V_{i}}\right)\left(\frac{V_{0} W_{0} Z_{0}}{U_{0}}-\frac{U_{i} V_{i} Z_{i}}{W_{i}}\right)\left(\frac{U_{0}}{V_{0} W_{0} Z_{0}}-\frac{U_{i} V_{i} W_{i}}{Z_{i}}\right)}\right] \\
& \times \frac{\left(\frac{v_{0} w_{0} z_{0}}{u_{0}}-u_{k} v_{k} w_{k} z_{k}\right)\left(\frac{v_{0} w_{0} z_{0}}{u_{0}}-\frac{w_{k} z_{k}}{u_{k} v_{k}}\right)\left(\frac{v_{0} w_{0} z_{0}}{u_{0}}-\frac{v_{k} z_{k}}{u_{k} w_{k}}\right)\left(\frac{v_{0} w_{0} z_{0}}{u_{0}}-\frac{u_{k} z_{k}}{v_{k} w_{k}}\right) \frac{u_{k} v_{k} w_{k}}{z_{k}}}{\left(1-u_{0}^{2}\right)\left(1-v_{0}^{2}\right)\left(1-w_{0}^{2}\right)\left(v_{0}^{2} w_{0}^{2}-u_{0}^{2}\right)} \\
& \times \frac{\prod_{i=0}^{k-1}\left[\left(1-u_{i}^{2}\right)\left(1-v_{i}^{2}\right)\left(1-w_{i}^{2}\right)\left(\frac{v_{0}^{2} w_{0}^{2} z_{0}^{2}}{u_{0}^{2}}-z_{i}^{2}\right)\right]}{\prod_{i=1}^{k}\left[\left(\frac{v_{0} w_{0} z_{0}}{u_{0}}-\frac{v_{i} w_{i} z_{i}}{u_{i}}\right)\left(\frac{v_{0} w_{0} z_{0}}{u_{0}}-\frac{u_{i} w_{i} z_{i}}{v_{i}}\right)\left(\frac{v_{0} w_{0} z_{0}}{u_{0}}-\frac{u_{i} v_{i} z_{i}}{w_{i}}\right)\left(\frac{u_{0}}{v_{0} w_{0} z_{0}}-\frac{u_{i} v_{i} w_{i}}{z_{i}}\right)\right]} \\
& =\frac{V_{0} W_{0} U_{0}^{3}}{Z_{0}^{3}} \frac{z_{0}^{3}}{v_{0} w_{0} u_{0}^{3}} \quad \\
& \times \prod_{i=1}^{n}\left[\frac{\left(1-u_{i}^{2}\right)\left(1-v_{i}^{2}\right)\left(1-w_{i}^{2}\right)\left(\frac{v_{0}^{2} w_{0}^{2} z_{0}^{2}}{u_{0}^{2}}-z_{i}^{2}\right)}{\left(\frac{v_{0} w_{0} z_{0}}{u_{0}}-\frac{v_{i} w_{i} z_{i}}{u_{i}}\right)\left(\frac{v_{0} w_{0} z_{0}}{u_{0}}-\frac{u_{i} w_{i} z_{i}}{v_{i}}\right)\left(\frac{v_{0} w_{0} z_{0}}{u_{0}}-\frac{u_{i} v_{i} z_{i}}{w_{i}}\right)\left(\frac{u_{0}}{v_{0} w_{0} z_{0}}-\frac{u_{i} v_{i} w_{i}}{z_{i}}\right)}\right] \\
& \times \sum_{j=0}^{n} \frac{\left(\frac{V_{0} W_{0} Z_{0}}{U_{0}}-U_{j} V_{j} W_{j} Z_{j}\right)\left(\frac{V_{0} W_{0} Z_{0}}{U_{0}}-\frac{W_{j} Z_{j}}{U_{j} V_{j}}\right)\left(\frac{V_{0} W_{0} Z_{0}}{U_{0}}-\frac{V_{j} Z_{j}}{U_{j} W_{j}}\right)\left(\frac{V_{0} W_{0} Z_{0}}{U_{0}}-\frac{U_{j} Z_{j}}{V_{j} W_{j}}\right)}{\left(1-U_{0}^{2}\right)\left(1-V_{0}^{2}\right)\left(1-W_{0}^{2}\right)\left(V_{0}^{2} W_{0}^{2}-U_{0}^{2}\right)}
\end{aligned}
$$




$$
\begin{aligned}
& \times \frac{U_{j} V_{j} W_{j}}{Z_{j}} \\
& \times \prod_{i=0}^{j-1}\left[\left(1-U_{i}^{2}\right)\left(1-V_{i}^{2}\right)\left(1-W_{i}^{2}\right)\left(\frac{V_{0}^{2} W_{0}^{2} Z_{0}^{2}}{U_{0}^{2}}-Z_{i}^{2}\right)\right. \\
& \left.\times\left(\frac{v_{0} w_{0} z_{0}}{u_{0}}-\frac{v_{n-i} w_{n-i} z_{n-i}}{u_{n-i}}\right)\left(\frac{v_{0} w_{0} z_{0}}{u_{0}}-\frac{u_{n-i} w_{n-i} z_{n-i}}{v_{n-i}}\right)\right] \\
& \times \frac{1}{\prod_{i=0}^{j}\left[\left(\frac{V_{0} W_{0} Z_{0}}{U_{0}}-\frac{U_{i} W_{i} Z_{i}}{U_{i}}\right)\left(\frac{V_{0} W_{0} Z_{0}}{U_{0}}-\frac{U_{i} W_{i} Z_{i}}{V_{i}}\right)\left(\frac{V_{0} W_{0} Z_{0}}{U_{0}}-\frac{U_{i} V_{i} Z_{i}}{W_{i}}\right)\left(\frac{U_{0}}{V_{0} W_{0} Z_{0}}-\frac{U_{i} V_{i} W_{i}}{Z_{i}}\right)\right]} \\
& \quad \times \frac{\prod_{i=0}^{j-1}\left[\left(\frac{v_{0} w_{0} z_{0}}{u_{0}}-\frac{u_{n-i} v_{n-i} z_{n-i}}{w_{n-i}}\right)\left(\frac{u_{0}}{v_{0} w_{0} z_{0}}-\frac{u_{n-i} v_{n-i} w_{n-i}}{z_{n-i}}\right)\right]}{\prod_{i=0}^{j}\left[\left(1-u_{1+n-i}^{2}\right)\left(1-v_{1+n-i}^{2}\right)\left(1-w_{1+n-i}^{2}\right)\left(\frac{v_{0}^{2} w_{0}^{2} z_{0}^{2}}{u_{0}^{2}}-z_{1+n-i}^{2}\right)\right]} .
\end{aligned}
$$

Transformation (2.4) can be proved by expanding the first product on the left hand side by using (2.3) (with $n$ replaced by $n-k$ ), interchanging the order of summations and evaluating the inner sum by using (2.3) once again and simplifying to get the right hand side of (2.4).

Transformation (2.4), on replacing $u_{i}, v_{i}, w_{i}, z_{i}, U_{i}, V_{i}, W_{i}, Z_{i}$ by $\sqrt{a} p^{i}$, $\sqrt{c} q^{i}, \sqrt{b} P^{i}, Q^{i}, \sqrt{A} \widetilde{p}^{i}, \sqrt{C} \widetilde{q}^{i}, \sqrt{B} \widetilde{P}^{i}, \widetilde{Q}^{i}$, respectively, reduces on some simplification to the following transformation of $q$-series involving eight independent bases:

$$
\begin{aligned}
\sum_{k=0}^{n} & \frac{\left(1-a p^{k} q^{k} P^{k} Q^{k}\right)\left(\frac{q^{k} P^{k} Q^{k}}{p^{k}}-c q^{2 k}\right)\left(1-b \frac{p^{k} P^{k}}{q^{k} Q^{k}}\right)\left(1-\frac{a}{b c} \frac{p^{k} Q^{k}}{q^{k} P^{k}}\right)}{(1-a)(1-c)(1-b)\left(1-\frac{a}{b c}\right)} \\
& \times \frac{\left(a ; p^{2}\right)_{k}\left(c ; q^{2}\right)_{k}\left(b ; P^{2}\right)_{k}\left(\frac{a}{b c} ; Q^{2}\right)_{k}}{\left(\frac{q P Q}{p} ; \frac{q P Q}{p}\right)_{k}\left(\frac{a}{c} \frac{p P Q}{q} ; \frac{p P Q}{q}\right)_{k}\left(\frac{a}{b} \frac{p q Q}{P} ; \frac{p q Q}{P}\right)_{k}\left(b c \frac{p q P}{Q} ; \frac{p q P}{Q}\right)_{k}} \\
& \times \frac{\left(\frac{\widetilde{q}^{-n} \widetilde{P}^{-n} \widetilde{Q}^{-n}}{\widetilde{p}^{-n}} ; \frac{\widetilde{q} \widetilde{P} \widetilde{Q}}{\widetilde{p}}\right)_{k}\left(\frac{C}{A} \frac{\widetilde{p}^{-n} \widetilde{P}^{-n} \widetilde{Q}^{-n}}{\widetilde{q}^{-n}} ; \frac{\widetilde{p} \widetilde{Q} \widetilde{Q}}{\widetilde{q}}\right)_{k}\left(\frac{B}{A} \frac{\widetilde{p}^{-n} \widetilde{q}^{-n} \widetilde{Q}^{-n}}{\widetilde{P}^{-n}} ; \frac{\widetilde{p} q \widetilde{Q}}{\widetilde{P}}\right)_{k}}{\left(\frac{\widetilde{p}^{-2 n}}{A} ; \widetilde{p}^{2}\right)_{k}\left(\frac{\widetilde{q}^{-2 n}}{C} ; \widetilde{q}^{2}\right)_{k}\left(\frac{\widetilde{P}^{-2 n}}{B} ; \widetilde{P}^{2}\right)_{k}\left(\frac{B C}{A} \widetilde{Q}^{-2 n} ; \widetilde{Q}^{2}\right)_{k}} \\
& \times\left(\frac{\widetilde{p}^{-n} \widetilde{q}^{-n} \widetilde{P}^{-n}}{B C \widetilde{Q}^{-n}} ; \frac{\widetilde{p} \widetilde{q} \widetilde{P}}{\widetilde{Q}}\right)_{k} \\
= & \frac{\left(a p^{2} ; p^{2}\right)_{n}\left(c q^{2} ; q^{2}\right)_{n}\left(b P^{2} ; P^{2}\right)_{n}\left(\frac{a Q^{2}}{b c} ; Q^{2}\right)_{n}}{\left(\frac{q P Q}{p} ; \frac{q P Q}{p}\right)_{n}\left(\frac{a}{c} \frac{p P Q}{q} ; \frac{p P Q}{q}\right)_{n}\left(\frac{a}{b} \frac{p q Q}{P} ; \frac{p q Q}{P}\right)_{n}\left(b c \frac{p q P}{Q} ; \frac{p q P}{Q}\right)_{n}}
\end{aligned}
$$




$$
\begin{aligned}
& \times \frac{\left(\frac{\widetilde{q} \widetilde{P}}{\widetilde{p}} ; \frac{\widetilde{q} \widetilde{P} \widetilde{Q}}{\widetilde{p}}\right)_{n}\left(\frac{A}{C} \frac{\widetilde{p} \widetilde{P} \widetilde{Q}}{\widetilde{q}} ; \frac{\widetilde{p} \widetilde{P} \widetilde{Q}}{\widetilde{q}}\right)_{n}\left(\frac{A}{B} \frac{\widetilde{p q} \widetilde{Q}}{\widetilde{P}} ; \frac{\widetilde{p} \widetilde{Q}}{\widetilde{P}}\right)_{n}\left(B C \frac{\widetilde{p} \widetilde{P}}{\widetilde{Q}} ; \frac{\widetilde{p} \widetilde{P}}{\widetilde{Q}}\right)_{n}}{\left(a \widetilde{p}^{2} ; \widetilde{p}^{2}\right)_{n}\left(C \widetilde{q}^{2} ; \widetilde{q}^{2}\right)_{n}\left(B \widetilde{P}^{2} ; \widetilde{P}^{2}\right)_{n}\left(\frac{A \widetilde{Q}^{2}}{B C} ; \widetilde{Q}^{2}\right)_{n}} \\
& \times \sum_{j=0}^{n} \frac{\left(1-A \widetilde{p}^{j} \widetilde{q}^{j} \widetilde{P}^{j} \widetilde{Q}^{j}\right)\left(\frac{\widetilde{q}^{j} \widetilde{P}^{j} \widetilde{Q}^{j}}{\widetilde{p}^{j}}-C \widetilde{q}^{2 j}\right)\left(1-\frac{B \widetilde{p}^{j} \widetilde{P}^{j}}{\widetilde{q}^{j} \widetilde{Q}^{j}}\right)\left(1-\frac{A}{B C} \frac{\widetilde{p}^{j} \widetilde{Q}^{j}}{\widetilde{q}^{j} \widetilde{P}^{j}}\right)}{(1-A)(1-C)(1-B)\left(1-\frac{A}{B C}\right)} \\
& \times \frac{\left(A ; \widetilde{p}^{2}\right)_{j}\left(C ; \widetilde{q}^{2}\right)_{j}\left(B ; \widetilde{P}^{2}\right)_{j}\left(\frac{A}{B C} ; \widetilde{Q}^{2}\right)_{j}}{\left(\frac{\widetilde{q} \widetilde{P} \widetilde{Q}}{\widetilde{p}} ; \frac{\widetilde{q} \widetilde{P} \widetilde{Q}}{\widetilde{p}}\right)_{j}\left(\frac{A}{C} \frac{\widetilde{p} \widetilde{P}}{\widetilde{q}} ; \frac{\widetilde{p} \widetilde{P}}{\widetilde{q}}\right)_{j}\left(\frac{A}{B} \frac{\widetilde{p} \widetilde{q}}{\widetilde{P}} ; \frac{\widetilde{p} \widetilde{Q}}{\widetilde{P}}\right)_{j}\left(B C \frac{\widetilde{p} \widetilde{P}}{\widetilde{Q}} ; \frac{\widetilde{p} \tilde{P}}{\widetilde{Q}}\right)_{j}} \\
& \times \frac{\left(\frac{q^{-n} P^{-n} Q^{-n}}{p^{-n}} ; \frac{q P Q}{P}\right)_{j}\left(\frac{c}{a} \frac{p^{-n} P^{-n} Q^{-n}}{q^{-n}} ; \frac{p P Q}{q}\right)_{j}\left(\frac{b}{a} \frac{p^{-n} q^{-n} Q^{-n}}{P^{-n}} ; \frac{p q Q}{P}\right)_{j}}{\left(\frac{p^{-2 n}}{a} ; p^{2}\right)_{j}\left(\frac{q^{-2 n}}{c} ; q^{2}\right)_{j}\left(\frac{P^{-2 n}}{b} ; P^{2}\right)_{j}\left(\frac{b c Q^{-2 n}}{a} ; Q^{2}\right)_{j}} \\
& \times\left(\frac{p^{-n} q^{-n} P^{-n}}{b c Q^{-n}} ; \frac{p q P}{Q}\right)_{j} .
\end{aligned}
$$

Transformation (2.5) is a generalization of Gasper's [4, Ex. 3.21] quadbasic transformation (from which Gasper deduces a transformation of a halfpoised ${ }_{10} \phi_{9}$ into another half-poised ${ }_{10} \phi_{9}[4$, Ex. 3.24]) to which it reduces on setting $Q=q, P=p, \widetilde{P}=\widetilde{p}, \widetilde{Q}=\widetilde{q}$ and then replacing $p^{2}, q^{2}, \widetilde{p}^{2}$ and $\widetilde{q}^{2}$ by $p, q, P$ and $Q$, respectively.

Next, we obtain an expansion of $\sum_{r=0}^{\infty} A_{r} B_{r}(x w)^{r} /(q ; q)_{r}$ terms of $q$-series having three independent bases. In the summation formula (2.2), setting $m=0, c=q^{-2 n}$, where $n$ is a non-negative integer and letting $d \rightarrow 1$, we get

$$
\begin{aligned}
& \sum_{j=0}^{n} \frac{\left(1-a p^{j} q^{j} P^{j} Q^{j}\right)\left(\frac{q^{j} P^{j} Q^{j}}{p^{j}}-q^{2 j-2 n}\right)\left(1-\frac{b p^{j} P^{j}}{q^{j} Q^{j}}\right)\left(1-\frac{a}{b} \frac{p^{j} Q^{j}}{q^{j-2 n} P^{j}}\right)}{(1-a)\left(1-q^{-2 n}\right)(1-b)\left(1-\frac{a}{b} q^{2 n}\right)} \\
& \quad \times \frac{\left(a ; p^{2}\right)_{j}\left(q^{-2 n} ; q^{2}\right)_{j}\left(b ; P^{2}\right)_{j}\left(\frac{a}{b} q^{2 n} ; Q^{2}\right)_{j}}{\left(\frac{q P Q}{p} ; \frac{q P Q}{p}\right)_{j}\left(\frac{a p P Q}{q^{1-2 n}} ; \frac{p P Q}{q}\right)_{j}\left(\frac{a}{b} \frac{p q Q}{P} ; \frac{p q Q}{P}\right)_{j}\left(\frac{b p q^{1-2 n} P}{Q} ; \frac{p q P}{Q}\right)_{j}}=\delta_{n, 0} .
\end{aligned}
$$

Replacing $a$ and $b$ by $a p^{r} q^{r} P^{r} Q^{r}$ and $b p^{r} q^{-r} P^{r} Q^{-r}$ respectively, where $r$ is a non-negative integer, setting $Q=\frac{p q}{P}$ and then replacing $p^{2}, q^{2}, P^{2}, n$ by $p, q, P, m$, respectively, we get

$$
\begin{aligned}
\delta_{m, 0}= & \sum_{j=0}^{m} \frac{\left(1-a p^{j+r} q^{j+r}\right)\left(1-b P^{j+r} q^{-j-r}\right)\left(1-\frac{a}{b} p^{r+j} q^{2 r+m} P^{-r-j}\right) q^{j}}{\left(1-a p^{r} q^{r}\right)\left(1-b P^{r} q^{-r}\right)\left(1-\frac{a}{b} p^{r} q^{2 r+m} P^{-r}\right)} \\
& \times \frac{\left(a p^{r} q^{r} ; p\right)_{j}\left(q^{-m} ; q\right)_{j}\left(b P^{r} q^{-r} ; P\right)_{j}\left(\frac{a}{b} p^{r} q^{2 r+m} P^{-r} ; \frac{p q}{P}\right)_{j}}{(q ; q)_{j}\left(a p^{1+r} q^{r+m} ; p\right)_{j}\left(\frac{a}{b} p^{r+1} q^{1+2 r} P^{-r-1} ; \frac{p q}{P}\right)_{j}\left(b q^{-m-r} P^{r+1} ; P\right)_{j}}
\end{aligned}
$$


But, we know that

$$
\begin{aligned}
& B_{r} x^{r} \\
& =\sum_{m=0}^{\infty} \frac{\left(1-\frac{a}{b} p^{r+m} q^{2 r+2 m} P^{-r-m}\right)\left(\frac{a}{b} p^{r} q^{2 r} P^{-r} ; \frac{p q}{P}\right)_{m}\left(a p q^{r} ; p\right)_{r}\left(b P q^{-r} ; P\right)_{r}}{\left(1-\frac{a}{b} p^{r} q^{2 r} P^{-r}\right)(q ; q)_{m}\left(a p q^{r+m} ; p\right)_{r}\left(b P q^{-m-r} ; P\right)_{r}} \\
& \quad \times\left\{q^{-r m} B_{r+m} C_{r, m} x^{r+m} \delta_{m, 0}\right\},
\end{aligned}
$$

where $\left\langle B_{r}\right\rangle$ and $\left\langle C_{r, m}\right\rangle$ are arbitrary sequences of complex numbers such that $C_{r, 0}=1$ for $r=0,1,2, \ldots$ Substituting for $\delta_{m, 0}$ from (2.6), interchanging the order of summation and setting $j=n-r$ and $m=n+k-r$, we get

$$
\begin{aligned}
B_{r} x^{r} & =\sum_{k=0}^{\infty} \sum_{n=r}^{\infty}(-)^{n}\left(1-a p^{n} q^{n}\right)\left(a p q^{r} ; p\right)_{n-1}\left(1-b P^{n} q^{-n}\right)\left(b P q^{-r} ; P\right)_{n-1} \\
& \times \frac{\left(1-\frac{a}{b} p^{n+k} q^{2 n+2 k} P^{-n-k}\right) x^{n+k}\left(q^{-n} ; q\right)_{r}}{\left(a p q^{n+k} ; p\right)_{n}\left(b P q^{-n-k} ; P\right)_{n}(q ; q)_{n}(q ; q)_{k}} \\
& \left\{\left(1-\frac{a}{b} p^{n} q^{n+k+r} P^{-n}\right)\left(\frac{a}{b} \frac{p^{n+1} q^{n+r+1}}{P^{n+1}} ; \frac{p q}{P}\right)_{k-1}\right. \\
& \left.\times\left(\frac{a}{b} p^{r+1} q^{n+k+r+1} P^{-r-1} ; \frac{p q}{P}\right)_{n-r-1} B_{n+k} C_{r, n+k-r} q^{n(r-k-n)+\frac{n^{2}}{2}+\frac{n}{2}}\right\} .
\end{aligned}
$$

Multiplying both sides by $\frac{A_{r} w^{r}}{(q ; q)_{r}}$ and summing from $r=0$ to $\infty$ and interchanging the order of summation on the right hand side, we get

$$
\begin{aligned}
& \sum_{r=0}^{\infty} A_{r} B_{r} \frac{(x w)^{r}}{(q ; q)_{r}} \\
& =\sum_{n=0}^{\infty} \frac{\left(1-a p^{n} q^{n}\right)\left(1-b P^{n} q^{-n}\right)(-x)^{n} q^{\left(\begin{array}{c}
n \\
2
\end{array}\right)+n}}{(q ; q)_{n}} \\
& \times \sum_{k=0}^{\infty} \frac{\left(1-\frac{a}{b} \frac{p^{n+k} q^{2 n+2 k}}{P^{n+k}}\right) x^{k} B_{n+k}}{(q ; q)_{k}\left(a p q^{n+k} ; p\right)_{n}\left(b P q^{-n-k} ; P\right)_{n}} \\
& \times \sum_{r=0}^{\infty} \frac{\left(q^{-n} ; q\right)_{r}\left(a p q^{r} ; p\right)_{n-1}\left(b P q^{-r} ; P\right)_{n-1}}{(q ; q)_{r}} \\
& \times\left\{\left(1-\frac{a}{b} \frac{p^{n} q^{n+k+r}}{P^{r}}\right)\left(\frac{a}{b} \frac{p^{n+1} q^{n+r+1}}{P^{n+1}} ; \frac{p q}{P}\right)_{k-1}\right. \\
& \left.\times\left(\frac{a}{b} \frac{p^{r+1} q^{n+k+r+1}}{P^{r+1}} ; \frac{p q}{P}\right)_{n-r-1}\right\} C_{r, n+k-r} w^{n} q^{n(r-k-n)} A_{r},
\end{aligned}
$$

which is a generalization of Gasper's bibasic expansion formula $[4,(3.7 .6)]$ to which it reduces on setting $P=p$. It may be noted that on setting $P=p$ the 
terms in $\{\cdots\}$ of the above expression combine to yield $\left(\frac{a}{b} q^{n+r+1} ; q\right)_{n+k-r-1}$ as in the bibasic expansion formula of Gasper [4, (3.7.6)].

\section{$\S 3$.}

All the summation formulae proved so far are for one generalization of (1.1), a very-well-poised $q$-series. We next derive a summation formula which gives the sum of a balanced series.

Let $\left\langle x_{i}\right\rangle,\left\langle y_{i}\right\rangle$ and $\left\langle z_{i}\right\rangle$ be arbitrary sequences and $a$ an indeterminate so that none of the terms in the denominators vanish and $m, n$ are non-negative integers. Then

$$
\begin{aligned}
& \sum_{k=-m}^{n} \frac{\left(1-\frac{y_{k}}{a z_{k}}\right)\left(1-\frac{x_{k}}{a z_{k}}\right) z_{k}}{\left(1-\frac{y_{0}}{a z_{0}}\right)\left(1-\frac{x_{0}}{a z_{0}}\right)} \frac{\prod_{i=0}^{k-1}\left[\left(1-x_{i}\right)\left(1-y_{i}\right)\right]}{\prod_{i=1}^{k}\left[\left(1-a z_{i}\right)\left(1-\frac{x_{i} y_{i}}{a z_{i}}\right)\right]} \\
& =\frac{\left(1-x_{0}\right)\left(1-y_{0}\right)}{a\left(1-\frac{y_{0}}{a z_{0}}\right)\left(1-\frac{x_{0}}{a z_{0}}\right)} \\
& \quad \times\left\{\prod_{i=1}^{n}\left[\frac{\left(1-x_{i}\right)\left(1-y_{i}\right)}{\left(1-a z_{i}\right)\left(1-\frac{x_{i} y_{i}}{a z_{i}}\right)}\right]-\prod_{i=-m}^{0}\left[\frac{\left(1-a z_{i}\right)\left(1-\frac{x_{i} y_{i}}{a z_{i}}\right)}{\left(1-x_{i}\right)\left(1-y_{i}\right)}\right]\right\} .
\end{aligned}
$$

For proving (3.1) we consider

$$
\tau_{k}=\prod_{i=1}^{k}\left[\frac{\left(1-x_{i}\right)\left(1-y_{i}\right)}{\left(1-a z_{i}\right)\left(1-\frac{x_{i} y_{i}}{a z_{i}}\right)}\right] .
$$

Then by straight forward calculations we find that

$$
\Delta \tau_{k}=a z_{k}\left(1-\frac{y_{k}}{a z_{k}}\right)\left(1-\frac{x_{k}}{a z_{k}}\right) \frac{\prod_{i=1}^{k-1}\left[\left(1-x_{i}\right)\left(1-y_{i}\right)\right]}{\prod_{i=1}^{k}\left[\left(1-a z_{i}\right)\left(1-\frac{x_{i} y_{i}}{z_{i}}\right)\right]},
$$

which on summing over $k$ from $-m$ to $n$, gives (3.1) after using (1.6).

In view of this it is natural to look for a telescoping proof of the $q$-Paff-Salschütz summation formula [4, (1.7.2)]

$$
S_{n} \equiv{ }_{3} \phi_{2}\left[\begin{array}{c}
a, b, q^{-n} ; q, q \\
c, \frac{a b}{c} q^{1-n}
\end{array}\right]=\frac{\left(\frac{c}{a}, \frac{c}{b} ; q\right)_{n}}{\left(c, \frac{c}{a b} ; q\right)_{n}} .
$$

To this end define for non-negative integers $n$ and $r$

$$
F(n, r)=\frac{\left(a, b, q^{-n} ; q\right)_{r} q^{r}}{\left(q, c, \frac{a b}{c} q^{1-n} ; q\right)_{r}}
$$




$$
\text { and } \quad G(n, r)=\frac{(a, b ; q)_{r+1}\left(q^{-n} ; q\right)_{r}}{\left(q, c, \frac{a b}{c} q^{1-n} ; q\right)_{r}} .
$$

Notice that $S_{0}=1, G(n, n+1)=G(0,-1)=0$. By straightforward calculations we can verify that

$$
\begin{aligned}
\left(1-\frac{c}{a} q^{n}\right)\left(1-\frac{c}{b} q^{n}\right) F(n, r)-(1 & \left.-c q^{n}\right)\left(1-\frac{c q^{n}}{a b}\right) F(n+1, r) \\
& -\frac{c}{a b} q^{n}[G(n, r)-G(n, r-1)]=0 .
\end{aligned}
$$

Summing over $r$ from 0 to $n+1$ and using $G(n, n+1)=0=G(0,-1)$, we get

$$
\left(1-\frac{c}{a} q^{n}\right)\left(1-\frac{c}{a} q^{n}\right) S_{n}=\left(1-c q^{n}\right)\left(1-\frac{c}{a b}\right) S_{n+1}
$$

which yields

$$
S_{n}=\frac{\left(\frac{c}{a}, \frac{c}{b} ; q\right)_{n}}{\left(c, \frac{c}{a b} ; q\right)_{n}} S_{0} .
$$

By using $S_{0}=1$, we get (3.2).

Acknowledgment. The authors wish to thank the referee for helpful comments.

\section{References}

[1] R.P. Agarwal, On the partial sums of series of hypergeometric type, Proc. Camb. Phil. Soc., 49 (1953), 441-445.

[2] W. Chu, Inversion techniques and combinatorial identities, Boll. U.M.I., 7(7) (1993), 737-760.

[3] G. Gasper, Summation, transformation, and expansion formulas for bibasic series, Trans. Amer. Math. Soc., 312 (1989), 257-277.

[4] G. Gasper and M. Rahman, Basic Hypergeometric Series, Cambridge University Press, 1990.

[5] _ An indefinite bibasic summation formula and some quadratic, cubic and quartic summation and transformation formulas, Canadian J. Math., 42 (1990), 1-27.

[6] V.K. Jain and A. Verma, Summation formulae for bibasic and polybasic hypergeometric series, J. Indian Math. Soc., 60 (1996).

Received January 2, 1998 and revised April 29, 1998. The first author was partially supported by an NSERC grant.

University of Alberta

Edmonton, Alberta T6G 2G1

CANADA

E-mail address: m.v.subbarao@ualberta.ca

UNIVERSITY OF ROORKEE

ROORKEE 247667, U.P.

INDIA 
E-mail address: maths@rurkiu.ernet.in 